\title{
Measuring Empathy among Dental Students and Interns: A Cross-Sectional Study from Dammam, Saudi Arabia
}

\author{
Muhammad Nazir ${ }^{D},{ }^{1}$ Muhanad Alhareky ${ }^{D},{ }^{1}$ Abdulrahman Alqahtani, ${ }^{2}$ \\ Leenah Alsulaimi, ${ }^{2}$ Reema Alotaibi, ${ }^{2}$ Najwa Yousef, ${ }^{2}$ Fatimah Abushal, ${ }^{2}$ \\ and Jehan Alhumaid ${ }^{1}{ }^{1}$ \\ ${ }^{1}$ Department of Preventive Dental Sciences, College of Dentistry, Imam Abdulrahman Bin Faisal University, P. O. Box 1982, \\ Dammam 31441, UAE \\ ${ }^{2}$ College of Dentistry, Imam Abdulrahman Bin Faisal University, P. O. Box 1982, Dammam 31441, UAE
}

Correspondence should be addressed to Muhammad Nazir; manazir@iau.edu.sa

Received 1 March 2021; Revised 21 April 2021; Accepted 22 April 2021; Published 27 April 2021

Academic Editor: Halim Issever

Copyright (c) 2021 Muhammad Nazir et al. This is an open access article distributed under the Creative Commons Attribution License, which permits unrestricted use, distribution, and reproduction in any medium, provided the original work is properly cited.

\begin{abstract}
Objective. To evaluate empathy and its related factors among undergraduate dental students and interns enrolled in a public dental college in Dammam, Saudi Arabia. Materials and Methods. This cross-sectional study used the Jefferson Scale of Empathy-Health Profession Students (JSE-HPS) version to determine empathy in 362 dental students and interns in Dammam, Saudi Arabia. The JSE-HPS is a 20-item 7-point Likert scale questionnaire, and its score ranges from 20 to 140 with high values indicating increased empathy. Influences of age, gender, class year, previous year's grade point average (GPA), educational attainment of parents, and monthly family income on empathy were evaluated. Results. Of 501 enrolled students and interns, 362 returned completed questionnaires, and the response rate of the study was 72\%. The sample's empathy score (JSPE-HPS scale) ranged from 70 to 129 with a mean of $96.75( \pm 13.76)$. Most participants believed that empathy is important for effective communication with patients (96.1\%) and can improve the provider-patient relationship (95.6\%). Females demonstrated a significantly higher mean empathy score $(99.98 \pm 14.01)$ than males $(92.72 \pm 12.35)(P<0.001)$. Similarly, the participants with high GPA $(98.06 \pm 13.69)$ had significantly greater mean empathy scores than those with low GPA $(94.84 \pm 13.68)(P=0.029)$. The mean empathy score increased significantly from junior students ( $3^{\text {rd }}$ and $4^{\text {th }}$ year students) to senior students $\left(5^{\text {th }}\right.$ and $6^{\text {th }}$ year students) and interns $(P=0.008)$. Multiple linear regression analysis showed that class year $(B=2.03, P=0.006)$ and GPA $(B=8.67, P=0.003)$ were significant factors associated with empathy. Conclusions. Empathy is important for effective patient communication and improved provider-patient relationship. Female gender, high GPA, and class years were associated with empathy. Empathy should be integrated into dental curricula for effective student learning and positive patient care outcomes.
\end{abstract}

\section{Introduction}

Empathy, an important phenomenon in patient and healthcare provider relationship, is the ability of an individual to stand in the shoes of another person to better understand his/her perspective or situation $[1,2]$. In healthcare, empathy is defined as "a predominantly cognitive attribute that involves an understanding of the patient's experiences, concerns, and perspectives, combined with a capacity to communicate this understanding and an intention to help" [3]. There are studies about an association between empathy among healthcare providers and positive clinical outcomes $[3,4]$. It was reported that diabetic patients treated by physicians with higher scores of empathy demonstrated significantly improved control of hemoglobin A1c than those patients who were treated by physicians with low empathy scores [3]. Similarly, diabetic patients who received treatment by physicians with a high empathy score showed significantly lower acute metabolic complications than the patients of physicians with moderate and low levels of empathy [4]. 
It is well documented that empathy is associated with improved enablement, increased participation and education, higher compliance, reduced emotional distress, and improved patient's quality of life [5-7]. On the other hand, lack of empathy can frustrate and disappoint patients who tend to avoid visiting healthcare providers [8]. In dental literature, empathy is known to reduce dental fear, improve the provider-patient relationship, enhance patient cooperation and compliance, and bring positive clinical outcomes and high patient satisfaction $[9,10]$. A study of students in five health sciences professions (dentistry, pharmacy, medicine, veterinary medicine, and nursing) found that the students of dentistry and nursing undergraduate programs had the highest mean empathy scores in the beginning of their first year [11]. There is also evidence about higher levels of empathy in female students compared with male students of dentistry and other healthcare professions [11-13].

Empathy scores among students can be increased and maintained significantly by giving them lectures and showing/discussing videos on empathy for improved patient care [14]. However, there are reports about the decline of empathy among medical and dental students $[5,10,11]$. For example, the first year dental students exhibited significantly higher levels of empathy than students of subsequent years [10]. Similarly, another study reported the highest mean empathy score in the first year $(117.23 \pm 14.19)$ and the lowest mean empathy score in postgraduate dental students $(108.77 \pm 9.12)$ [15].

There is unquestionable importance of empathy in clinical practice; however, its various aspects have not been fully investigated in dentistry. Therefore, it is important to evaluate the influence of gender, grade point average (GPA), class year, and socioeconomic status factors on the perception of empathy. The understanding of empathy among students and interns can be used to improve the dental curricula and ensure a high quality of patient care. The current study aimed to evaluate empathy and its related factors among undergraduate dental students and interns enrolled in a public dental college in Dammam, Saudi Arabia.

\section{Materials and Methods}

2.1. Participants. This cross-sectional study was conducted on dental students and interns at the College of Dentistry, Imam Abdulrahman Bin Faisal University, Dammam. The students spend one year in learning basic sciences and language courses at the preparatory college at the university, and they join their undergraduate dentistry program in second year. That is why the students in the first year were excluded from the study. The second year students were also excluded from the study because they are not exposed to clinical training and patient care. Therefore, the students receiving clinical training and providing patient treatment $\left(3^{\text {rd }}\right.$ year through $6^{\text {th }}$ years at the college) and interns were eligible to participate in the study. The recruitment of participants was carried out by using a census sample of all $3^{\text {rd }}-6^{\text {th }}$ year dental students and interns. There are 419 students in third, fourth, fifth, and sixth year classes and 82 interns $(N 419+82=501)$. Those students and interns who provided their verbal informed consent received a self-administered paper-based questionnaire.

2.2. Study Instrument. The questionnaire can be broadly divided into three sections. The first section of the questionnaire included demographic profiles of participants such as age, gender, class year, previous year grade point average (GPA), educational attainment of parents, and monthly family income. The second section included the Jefferson Scale of Physician Empathy-Health Profession Students (JSPE-HPS) version adapted from Hojat et al., 2001 [16]. There are 20 items in the scale which assess health profession students' self-reported empathy. Half of these items are positively worded statements and the other half are negatively worded statements. A 7-point Likert scale ( $1=$ strongly disagree; 7 = strongly agree) is used for these items. The score of the JSPE-HPS version ranges 20-140. Previous studies performed the psychometric analysis of the JSPE-HPS version and showed that the instrument is valid and reliable to evaluate empathy levels among health profession students $[2,17]$. The third section of the questionnaire included six questions about the importance of empathy in improving patient communication, dentist-patient relationship, positive treatment outcomes, quality of dental care, patient satisfaction, and reducing rates of patient litigation. The questionnaire was also reviewed to evaluate its appropriateness from the cultural point of view.

2.3. Ethical Considerations. Aims of the study including its details were discussed with study participants. They were informed about their right to voluntary participation in the survey. The privacy and confidentiality of their responses were ensured by an anonymous survey. Explanations about the items of the questionnaire were provided to the study participants. Self-administered paper-based questionnaires were administered in classes after students finish their lectures. Dental interns received the questionnaire in their clinics in the dental hospital. Two subsequent visits were made if interns were unable to provide their responses in the first visit due to their clinical commitments. The scientific research unit (ethics committee) at the college provided ethical approval of the study (EA 202016) on 10/11/2019. Ethical standards of the Helsinki Declaration were followed during the study.

2.4. Statistical Analysis. Data were entered in MS Excel and then transported and stored in the SPSS version 22 (IBM, Armonk, NY, USA). Descriptive statistics included means, standard deviations, frequencies, and proportions. The first 10 negatively worded items were reverse coded; then, the total empathy score for each participant was calculated by adding the responses to 20 items of the JSPE-HPS scale, and finally, the mean empathy score of the sample was calculated. Analytical statistics were performed using the independentsample Student's $t$-test and one-way ANOVA test. These tests compared mean empathy scores between male and 
female students and among students from different years of study, parental education, and monthly family income (low income $\leq 5000 \mathrm{SAR} /$ month, middle income 5001-20000 $\mathrm{SAR} /$ month, and high income $>20000 \mathrm{SAR} / \mathrm{month})$. One thousand SAR is equivalent to \$US266. The bivariate analysis included using the Pearson's correlation test. Multiple linear regression analysis was also performed to evaluate the factors that independently correlated with the empathy score. A $P$ value $<0.05$ was considered statistically significant.

\section{Results}

Three hundred and sixty-two students and interns participated in the study with $44.5 \%$ of males and $55.5 \%$ of females. The response rate was $362 / 501=72 \%$. Most participants (59.4\%) had high GPA (4-4.9) and had college/university educated fathers (65.5\%) and mothers (55.2\%). Threequarters of the participants reported that empathy was taught in their undergraduate course. The empathy score (JSPE-HPS scale) of the sample ranged from 70 to 129 with a mean of $96.75( \pm 13.76)$. Females demonstrated a significantly higher mean empathy score $(99.98 \pm 14.01)$ than males $(92.72 \pm 12.35) \quad(P<0.001)$. The mean empathy score increased significantly from junior students $\left(3^{\text {rd }}\right.$ and $4^{\text {th }}$ year students) to senior students $\left(5^{\text {th }}\right.$ and $6^{\text {th }}$ year students) and interns $(P=0.008)$. The mean empathy score was significantly higher in participants with high GPA $(98.06 \pm 13.69)$ than those with a low GPA $(94.84 \pm 13.68)(P=0.029)$. The empathy score rose with increasing parental educational levels and monthly family incomes; however, differences in mean scores were not statistically significant. The participants who said empathy was taught in the course had a higher mean empathy score $(97.15 \pm 14.09)$ than those who denied learning empathy $(95.6 \pm 12.73)(P=0.351)$ (Table 1$)$.

Nearly $96.1 \%$ of the participants believed that empathy is important for effective communication with patients, and 95.6\% believed that having empathy can improve the provider-patient relationship. The participants who believed in the role of empathy in effective communication, improved provider-patient relationship, positive treatment outcomes, enhanced quality of patient care, and improved patient satisfaction that demonstrated significantly higher mean empathy scores than those who did not believe in these characteristics (Table 2).

Bivariate linear regression analyses showed that class year significantly correlated with the empathy score $(r=0.15$, $P=0.017)$. Similarly, there was a significant correlation between GPA and the empathy score $(r=0.22, P=0.001)$ (Table 3).

In the multiple linear regression model, class year $(B$ 2.03, $P=0.006)$ and GPA $(B 8.67, P=0.003)$ remained significant factors associated with empathy among study participants. As the class year increases by one unit, empathy among study participants increases by a factor of 2.03 . Similarly, a unit increase in GPA increases empathy by a factor of 8.67. On the other hand, parental education and monthly family income showed no significant relationship with empathy (Table 4).

\section{Discussion}

The study aimed to assess levels of empathy and its influencing factors among dental students and interns. It was found that the female gender, high GPA, and senior class years/internship were significantly related to high empathy in our sample. The mean empathy score in our study was $96.75 \pm 13.76$ which is higher than that reported in a recent study of Saudi dental students by Naguib et al. in Jeddah who showed that the mean empathy score was $84.84 \pm 11.28$ [18]. Dental literature shows varying levels of empathy in dental students in different parts of the world. Based on the Jefferson Scale of Empathy-Health Profession Students, previous studies reported high mean empathy scores in American dental students (117.71 \pm 14.06$)$ [10], Nigerian dental students (104.01 \pm 19.64$)$ [19], and Pakistani dental students (101.15 \pm 13.73$)$ [20]. Conversely, low mean empathy scores (78-90) were reported in Malaysian, Indian, Iranian, Polish, and British dental students [21-25]. These variations in empathy levels in dental students in different countries may be attributed to the differences in cultures, traditions, religion, emotional status, psychological characteristics, and prosocial behaviors $[19,26]$.

The researchers from three universities in the U.S. collected data on empathy among 104,365 adults from 63 countries and revealed that Saudi Arabia was the second most empathetic country, whereas the U.S. was ranked the seventh most empathetic country in the world. The authors reported that countries with higher empathy had higher conscientiousness, collectivism, sociability, self-esteem, well-being, emotionality, and prosocial behavior such as volunteerism and help [26]. Despite, Saudi Arabia was ranked among the countries with the highest empathy score globally; however, the empathy score in Saudi dental students was lower than reported in dental students of some other countries $[10,19,20]$. The differences in study design and the measurement tool for the evaluation of empathy may account for inconsistencies in empathy levels in adult populations and dental students.

Globally, women exhibit higher total empathy, empathetic concern, and perspective-taking than men [26]. Literature shows that Malaysian and Indian male dental students demonstrated higher mean empathy scores than female students $[23,24]$. However, consistent with previous research in dental students $[10,15,18,20]$, females had significantly higher empathy scores than males in the current study. Similarly, in the majority of studies on medical and other health profession students, the higher empathy score was reported in females than males $[2,12,13,27]$. The caring nature and emotionally sensitive characteristics of females might be the reason for more empathetic behavior in females than their male counterparts $[20,28]$. Factors such as social learning, genetic prediction, and the evolutionary process may also be responsible for gender differences regarding empathy [28].

Our study showed a significant increase in empathy scores from the third year to sixth year and internship. A recent study of Saudi dental students reported higher empathy scores in fifth- and sixth-year students than third- and 
TABLE 1: Association between various sociodemographic factors and empathy among participants.

\begin{tabular}{|c|c|c|c|c|c|c|}
\hline \multirow[t]{2}{*}{ Variables } & \multirow[t]{2}{*}{$N(\%), N=362$} & \multirow[t]{2}{*}{ Mean empathy score } & \multirow[t]{2}{*}{ SD } & \multicolumn{2}{|c|}{$\begin{array}{c}95 \% \text { confidence } \\
\text { interval }\end{array}$} & \multirow[t]{2}{*}{$P$ value } \\
\hline & & & & Lower & Upper & \\
\hline \multicolumn{7}{|l|}{ Gender } \\
\hline Male & $161(44.5)$ & 92.72 & 12.35 & 90.82 & 94.67 & \multirow[t]{2}{*}{$<0.001$} \\
\hline Female & $201(55.5)$ & 99.98 & 14.01 & 97.93 & 101.76 & \\
\hline \multicolumn{7}{|l|}{ Class year } \\
\hline 3rd year & $87(24)$ & 94.73 & 14.71 & 91.6 & 97.87 & \multirow[t]{5}{*}{0.008} \\
\hline 4th year & $84(23.2)$ & 93.24 & 13.54 & 90.3 & 96.18 & \\
\hline 5 th year & $78(21.5)$ & 99.79 & 13.98 & 96.64 & 102.95 & \\
\hline 6 th year & $86(23.8)$ & 98.91 & 12.06 & 96.32 & 101.49 & \\
\hline Interns & $27(7.5)$ & 98.55 & 13.16 & 93.35 & 103.76 & \\
\hline \multicolumn{7}{|l|}{ Grade point average } \\
\hline High (4-4.9) & $215(59.4)$ & 98.06 & 13.69 & 96.12 & 99.85 & \multirow[t]{2}{*}{0.029} \\
\hline Low (3-3.9) & $147(40.6)$ & 94.84 & 13.68 & 92.64 & 97.11 & \\
\hline \multicolumn{7}{|l|}{ Mother's education } \\
\hline No formal education & $24(6.6)$ & 92.17 & 13.47 & 86.48 & 97.85 & \multirow[t]{3}{*}{0.198} \\
\hline School education & $138(38.1)$ & 97.64 & 14.14 & 95.26 & 100.02 & \\
\hline College/university education & $200(55.2)$ & 96.69 & 13.48 & 94.81 & 98.57 & \\
\hline \multicolumn{7}{|l|}{ Father's education } \\
\hline No formal education & $10(2.8)$ & 89.9 & 10.79 & 82.18 & 97.62 & \multirow[t]{3}{*}{0.271} \\
\hline School education & $115(31.8)$ & 97.22 & 14.1 & 94.61 & 99.82 & \\
\hline College/university education & $237(65.5)$ & 96.82 & 13.67 & 95.07 & 98.57 & \\
\hline \multicolumn{7}{|l|}{ Monthly family income } \\
\hline Low income & $14(3.9)$ & 90.93 & 10.69 & 84.75 & 97.1 & \multirow[t]{4}{*}{0.296} \\
\hline Middle income & $138(38.1)$ & 97.62 & 13.69 & 95.32 & 99.93 & \\
\hline High income & $128(35.4)$ & 97.16 & 14.03 & 94.71 & 99.62 & \\
\hline I do not know & $82(22.7)$ & 95.65 & 13.82 & 92.61 & 98.68 & \\
\hline \multicolumn{7}{|l|}{ Empathy taught in the course } \\
\hline Yes & $270(74.6)$ & 97.15 & 14.09 & 95.51 & 98.84 & \multirow[t]{2}{*}{0.351} \\
\hline No & $92(25.4)$ & 95.6 & 12.73 & 93.11 & 98.11 & \\
\hline
\end{tabular}

TABle 2: Association between patient care factors and empathy among participants.

\begin{tabular}{|c|c|c|c|c|c|c|}
\hline \multirow[t]{2}{*}{ Variables } & \multirow[t]{2}{*}{$N(\%)$} & \multirow[t]{2}{*}{$\begin{array}{l}\text { Mean empathy } \\
\text { score }\end{array}$} & \multicolumn{3}{|c|}{$\begin{array}{c}95 \% \\
\text { confidence } \\
\text { interval }\end{array}$} & \multirow[t]{2}{*}{$P$ value } \\
\hline & & & & Lower & Upper & \\
\hline \multirow{2}{*}{$\begin{array}{l}\text { (1) Do you believe that empathy is important for effective } \\
\text { communication with patients? }\end{array}$} & $\begin{array}{c}\text { Yes: } 348 \\
(96.1)\end{array}$ & 97.24 & 13.69 & 95.77 & 98.7 & \multirow[t]{2}{*}{$<0.001$} \\
\hline & No: 14 (3.9) & 83.64 & 7.33 & 79.77 & 87.89 & \\
\hline \multirow{2}{*}{$\begin{array}{l}\text { (2) Do you believe that empathy can help lower the rates of patient } \\
\text { litigation? }\end{array}$} & $\begin{array}{c}\text { Yes: } 321 \\
(88.7)\end{array}$ & 96.98 & 14.03 & 95.44 & 98.57 & \multirow{2}{*}{0.387} \\
\hline & $\begin{array}{c}\text { No: } 41 \\
(11.3)\end{array}$ & 95 & 11.39 & 91.54 & 98.64 & \\
\hline \multirow{2}{*}{$\begin{array}{l}\text { (3) Do you believe that having empathy can improve healthcare provider } \\
\text { and patient relationship? }\end{array}$} & $\begin{array}{c}\text { Yes: } 346 \\
(95.6)\end{array}$ & 97.22 & 13.69 & 95.79 & 98.82 & \multirow[t]{2}{*}{0.003} \\
\hline & No: $16(4.4)$ & 86.75 & 11.48 & 81.6 & 93.1 & \\
\hline \multirow[t]{2}{*}{ (4) Do you believe that empathy can lead to positive treatment outcomes? } & $\begin{array}{c}\text { Yes: } 337 \\
(93.1)\end{array}$ & 97.65 & 13.69 & 96.13 & 99.1 & \multirow[t]{2}{*}{$<0.001$} \\
\hline & No: 25 (6.9) & 84.6 & 7.54 & 81.83 & 87.68 & \\
\hline \multirow{2}{*}{$\begin{array}{l}\text { (5) Do you believe that empathy can improve the quality of patient dental } \\
\text { care? }\end{array}$} & $\begin{array}{c}\text { Yes: } 332 \\
(91.7)\end{array}$ & 97.21 & 13.95 & 95.63 & 98.73 & \multirow[t]{2}{*}{0.034} \\
\hline & No: $30(8.3)$ & 91.67 & 10.36 & 88.18 & 95.36 & \\
\hline \multirow{2}{*}{$\begin{array}{l}\text { (6) Do you believe that empathy is important for achieving patient } \\
\text { satisfaction? }\end{array}$} & $\begin{array}{c}\text { Yes: } 334 \\
(92.3)\end{array}$ & 97.24 & 13.9 & 95.72 & 98.74 & \multirow[t]{2}{*}{0.02} \\
\hline & No: $28(7.7)$ & 90.96 & 10.7 & 87.03 & 94.92 & \\
\hline
\end{tabular}


TABLE 3: Bivariate linear regression analyses: correlation between sociodemographic factors and empathy score in study participants.

\begin{tabular}{lcc}
\hline Correlation & Pearson's correlation coefficient (95\% CI) & $P$ value \\
\hline Class year and empathy score & $0.15(0.04,0.27)$ & 0.017 \\
GPA and empathy score & $0.22(0.01,0.34)$ & 0.001 \\
Mother's education and empathy score & $0.05(-0.08,0.18)$ & 0.459 \\
Father's education and empathy score & $-0.02(-0.15,0.11)$ & 0.810 \\
Monthly family income and empathy score & $-0.01(-0.14,0.12)$ & 0.910 \\
\hline
\end{tabular}

TABLE 4: Multivariable linear regression analyses: correlation between sociodemographic factors and empathy among study participants.

\begin{tabular}{|c|c|c|c|c|c|}
\hline \multirow{3}{*}{ Independent variables } & \multicolumn{4}{|c|}{ Coefficients } & \multirow{3}{*}{$P$ value } \\
\hline & \multicolumn{2}{|c|}{ Unstandardized coefficients } & \multicolumn{2}{|c|}{ Standardized coefficients } & \\
\hline & $B(95 \%$ confidence interval $)$ & SE & B coefficient & $t$ & \\
\hline Class year & $2.03(0.57,3.47)$ & 0.74 & 0.18 & 2.76 & 0.006 \\
\hline GPA & $8.67(2.97,14.41)$ & 2.89 & 0.19 & 2.99 & 0.003 \\
\hline Mother's education & $0.803(-2.179,3.800)$ & 1.517 & 0.036 & 0.529 & 0.597 \\
\hline Father's education & $-0.681(-4.005,2.625)$ & 1.683 & -0.027 & -0.405 & 0.686 \\
\hline Monthly family income & $-0.661(-4.005,2.625)$ & 1.111 & -0.040 & -0.595 & 0.552 \\
\hline
\end{tabular}

fourth-year students; however, the difference was not significant [18]. Similar trends were observed among Polish dental students who showed increased empathy scores from the first/second year to the fifth year but with no significant difference [25]. Conversely, previous studies showed significantly higher empathy scores in first-year students than students in the subsequent year of the dentistry program $[10,15]$. The increase in the empathy score from early years to later years of the undergraduate program is possibly related to increased exposure to clinical training and greater interaction with patients and clinical practice $[18,25]$.

In a descriptive cohort study, Carr et al. demonstrated significant correlations between the GPA of students and their workplace performance as junior doctors reflected both in clinical management and communication subscales [29]. It is also known that empathy is associated with clinical competence and communication [27, 30]. Therefore, it was assumed that those participants who have high GPA would be more empathetic than those with low GPA. Our findings confirmed this, and the empathy score was significantly higher in participants with a high GPA than those with a low GPA. In addition, GPA was a significant predictor of empathy after controlling for class year, parental education levels, and monthly family income. However, these findings contrast with the literature on empathy among medical students where Hojat et al. found no significant relationship between empathy and performance in the Medical College Admission Test (MCAT) and the US Medical Licensing Examinations (USMLE) [27].

The development of empathy behavior is important for dental students and the dental profession because of improved clinical outcomes associated with high levels of empathy $[3,28]$. In the studies of medical students, the empathy score in students was significantly associated with faculty's rating of clinical competence and communication scores of students $[27,30]$. Hence, there is a greater emphasis on empathy among dental students which is evident from the recent surge in the literature on dental education $[18-20,25,31]$. In our study, a vast majority of participants believed in the importance of empathy in effective communication with patients and improved provider-patient relationship. Similarly, those who believed that empathy is important for effective communication and improved provider-patient relationship exhibited significantly higher empathy scores than those who did not believe in the role of empathy in patient communication and provider-patient relationship.

Parental socioeconomic influences are known to affect learning performance and academic growth which can lead to clinical performance in students $[29,32]$. The current study investigated the influence of parental education and monthly family income levels on empathy among participants. It was hypothesized that participants with low socioeconomic status would demonstrate low empathy. Our findings confirmed that the lower empathy score was obtained from the participants with low parental education and low family income than those with high parental education and income levels, although differences in the mean scores of empathy were not statistically significant.

The use of a census sample of participants in the current study provided reliable evidence on empathy in dental education. However, there are certain limitations to the study. The evaluation of empathy through self-reported data may not truly reflect the level of empathy that participants may actually demonstrate in dental practice. The data collected from one dental college can limit the external validity of the study. In addition, the cross-sectional design is limited in establishing cause and effect relationships. For instance, it is not possible to ascertain if increased GPA can result in an increased empathy score or increased empathy can lead to a high GPA. Given the important role of empathy in clinical practice, the current study is expected to stimulate further research among students, faculty, and dental practitioners. In the future, a prospective multicenter study design should be used to assess empathy and its relationship with different variables among faculty and students. 


\section{Conclusions}

A vast majority of participants believed in the importance of empathy in effective communication with patients and improving the provider-patient relationship. The study showed significantly increased empathy in females than males. Similarly, the participants with high GPA were significantly more empathetic than the participants with low GPA. Additionally, GPA independently and significantly predicted empathy levels. Junior students demonstrated lower empathy which increased with advancing class years. However, socioeconomic status variables showed no significant influence on the empathy score. Dental academia may use the study findings when designing undergraduate curricula to enrich student learning and improve patient care. The results of the study may also have implications for the selection/admission criteria for prospective dental students.

\section{Data Availability}

The SPSS data file used to support the findings of this study is available from the corresponding author upon request.

\section{Conflicts of Interest}

The authors declare that there are no conflicts of interest.

\section{References}

[1] F. Derksen, J. Bensing, and A. Lagro-Janssen, "Effectiveness of empathy in general practice: a systematic review," British Journal of General Practice, vol. 63, no. 606, pp. e76-e84, 2013.

[2] B. Williams, T. Brown, L. McKenna et al., "Empathy levels among health professional students: a cross-sectional study at two universities in Australia," Advances in Medical Education and Practice, vol. 5, pp. 107-113, 2014.

[3] M. Hojat, D. Z. Louis, F. W. Markham, R. Wender, C. Rabinowitz, and J. S. Gonnella, "Physicians' empathy and clinical outcomes for diabetic patients," Academic Medicine, vol. 86, no. 3, pp. 359-364, 2011.

[4] S. D. Canale, D. Z. Louis, V. Maio et al., "The relationship between physician empathy and disease complications," Academic Medicine, vol. 87, no. 9, pp. 1243-1249, 2012.

[5] M. Neumann, F. Edelhäuser, D. Tauschel et al., "Empathy decline and its reasons: a systematic review of studies with medical students and residents," Academic Medicine, vol. 86, no. 8, pp. 996-1009, 2011.

[6] S. S. Kim, S. Kaplowitz, and M. V. Johnston, "The effects of physician empathy on patient satisfaction and compliance," Evaluation \& the Health Professions, vol. 27, no. 3, pp. 237251, 2004.

[7] S. Price, S. W. Mercer, and H. MacPherson, "Practitioner empathy, patient enablement and health outcomes: a prospective study of acupuncture patients," Patient Education and Counseling, vol. 63, no. 1-2, pp. 239-245, 2006.

[8] F. Derksen, T. C. Olde Hartman, A. van Dijk, A. Plouvier, J. Bensing, and A. Lagro-Janssen, "Consequences of the presence and absence of empathy during consultations in primary care: a focus group study with patients," Patient Education and Counseling, vol. 100, no. 5, pp. 987-993, 2017.
[9] C. Yarascavitch, G. Regehr, B. Hodges, and D. A. Haas, "Changes in dental student empathy during training," Journal of Dental Education, vol. 73, no. 4, pp. 509-517, 2009.

[10] J. J. Sherman and A. Cramer, "Measurement of changes in empathy during dental school," Journal of Dental Education, vol. 69, no. 3, pp. 338-345, 2005.

[11] P. Nunes, S. Williams, B. Sa, and K. Stevenson, "A study of empathy decline in students from five health disciplines during their first year of training," International Journal of Medical Education, vol. 2, pp. 12-17, 2011.

[12] S. K. Fields, P. Mahan, P. Tillman, J. Harris, K. Maxwell, and M. Hojat, "Measuring empathy in healthcare profession students using the Jefferson Scale of Physician Empathy: health provider - student version," Journal of Interprofessional Care, vol. 25, no. 4, pp. 287-293, 2011.

[13] H. U. Kataoka, N. Koide, K. Ochi, M. Hojat, and J. S. Gonnella, "Measurement of empathy among Japanese medical students: psychometrics and score differences by gender and level of medical education," Academic Medicine, vol. 84, no. 9, pp. 1192-1197, 2009.

[14] M. Hojat, D. Axelrod, J. Spandorfer, and S. Mangione, "Enhancing and sustaining empathy in medical students," Medical Teacher, vol. 35, no. 12, pp. 996-1001, 2013.

[15] V. Aggarwal, R. Garg, N. Goyal et al., "Exploring the missing link - empathy among dental students: an institutional crosssectional survey," Dental Research Journal, vol. 13, no. 5, pp. 419-423, 2016.

[16] M. Hojat, S. Mangione, T. J. Nasca et al., "The Jefferson Scale of Physician Empathy: development and preliminary psychometric data," Educational and Psychological Measurement, vol. 61, no. 2, pp. 349-365, 2001.

[17] B. Williams, T. Brown, M. Boyle, and S. Dousek, "Psychometric testing of the Jefferson scale of empathy health profession students' version with Australian paramedic students," Nursing \& Health Sciences, vol. 15, no. 1, pp. 45-50, 2013.

[18] G. H. Naguib, A. M. Sindi, M. H. Attar, E. N. Alshouibi, and M. T. Hamed, "A cross-sectional study of empathy among dental students at King Abdulaziz University," Journal of Dental Education, vol. 84, no. 1, pp. 22-26, 2020.

[19] P. O. Ameh, O. G. Uti, and O. O. Daramola, "Empathy among dental students in a Nigerian institution," European Journal of Dental Education, vol. 23, no. 2, pp. 135-142, 2019.

[20] M. Q. Javed, "The evaluation of empathy level of undergraduate dental students in Pakistan: a cross-sectional study," Journal of Ayub Medical College Abbottabad, vol. 31, pp. 402-406, 2019.

[21] F. Khalifesoltani, F. Nilchian, A. Yosefi et al., "Empathy levels in undergraduate and postgraduate dental students in Isfahan Faculty of Dentistry in 2011," Journal of Isfahan Dental School, vol. 7, no. 5, pp. 753-762, 2012.

[22] A. Beattie, J. Durham, J. Harvey, J. Steele, and S. McHanwell, "Does empathy change in first-year dental students?" European Journal of Dental Education, vol. 16, no. 1, pp. e111-e116, 2012.

[23] V. Kalyan, S. Manjula, T. Padma, K. V. N. R. Pratap, P. Vineela, and S. Varma, "Assessment of empathy among clinical dental students in a teaching dental institution in Telangana State, India," Journal of Indian Association of Public Health Dentistry, vol. 15, no. 2, p. 162, 2017.

[24] M. G. Babar, H. Omar, L. P. Lim et al., "An assessment of dental students' empathy levels in Malaysia," International Journal of Medical Education, vol. 4, p. 223, 2013.

[25] K. Mocny-Pachonska, P. Lanowy, A. Trzcionka et al., "Gender related changes of empathy level among Polish dental 
students over the course of training," Medicine (Baltimore), vol. 99, Article ID e18470, 2020.

[26] W. J. Chopik, E. O’Brien, and S. H. Konrath, "Differences in empathic concern and perspective taking across 63 countries," Journal of Cross-Cultural Psychology, vol. 48, no. 1, pp. 23-38, 2017.

[27] M. Hojat, J. S. Gonnella, S. Mangione et al., "Empathy in medical students as related to academic performance, clinical competence and gender," Medical Education, vol. 36, no. 6, pp. 522-527, 2002.

[28] M. Hojat, J. DeSantis, S. C. Shannon et al., "The Jefferson scale of empathy: a nationwide study of measurement properties, underlying components, latent variable structure, and national norms in medical students," Advances in Health Sciences Education, vol. 23, no. 5, pp. 899-920, 2018.

[29] S. E. Carr, A. Celenza, I. B. Puddey et al., "Relationships between academic performance of medical students and their workplace performance as junior doctors," BMC Medical Education, vol. 14, p. 157, 2014.

[30] R. S. Casas, Z. Xuan, A. H. Jackson, L. E. Stanfield, N. C. Harvey, and D. C. Chen, "Associations of medical student empathy with clinical competence," Patient Education and Counseling, vol. 100, no. 4, pp. 742-747, 2017.

[31] M. Carvajal, S. López, P. Sarabia-Alvarez et al., "Empathy levels of dental faculty and students: a survey study at an academic dental institution in Chile," Journal of Dental Education, vol. 83, no. 10, pp. 1134-1141, 2019.

[32] M. B. Dube and P. R. Mlotshwa, "Factors influencing enrolled nursing students' academic performance at a selected private nursing education institution in KwaZulu-Natal," Curationis, vol. 41, no. 1, pp. e1-e7, 2018. 